\title{
Recurrent ulcer after vagotomy and pyloroplasty: the $x$-ray appearances and their value in diagnosis
}

\author{
J. ALEXANDER WilliamS ${ }^{1}$ AND D. K. M. TOYE \\ From the General Hospital, Birmingham
}

SUMMARY Barium meal studies have shown evidence of a recurrent ulcer or of stenosis in 12 out of 24 patients with recurrent dyspepsia after vagotomy and pyloroplasty. These 12 patients have been subsequently proved to have had recurrences and all but one are now cured by further surgery. Barium meals in 12 patients show no evidence of recurrence or stenosis, and follow-up clinical studies suggest that they do not have recurrent ulcer.

Radiological studies appear to have great value in the interpretation of recurrent dyspeptic symptoms after vagotomy and pyloroplasty.

In a study of 250 consecutive patients with peptic ulcer treated by vagotomy and pyloroplasty and all subjected to regular yearly review, particular emphasis has been placed on the evaluation of recurrent or residual symptoms. So far 24 patients have complained of recurrent dyspepsia sufficient to warrant investigation and all these have had barium meal $x$-ray examinations. This paper reports the radiological findings in those patients proved to have had recurrent ulcer, and attempts to assess the value of radiology in managing the patient suspected of having a recurrence.

\section{Radiological Technique}

The technique of examination has been described in detail elsewhere (Toye, Hutton, and Williams, 1970) but will be briefly summarized here. Other possible causes of recurrent dyspepsia are first excluded: these are gallstones, hiatus hernia, and oesophageal reflux. The barium study is then directed towards the demonstration of gastric outlet obstruction or recurrent ulceration. The presence of resting contents in the stomach and delay in the passage of barium through the pyloroplasty area indicates the

${ }^{1}$ Member of the external staff, Medical Research Council. presence of gastric outlet obstruction. Particular attention is next paid to the mucosal pattern in the pyloroplasty channel and compression and air/barium contrast studies are made. It is also important to distend and then empty the pyloroplasty channel to demonstrate the flexibility and muscular movements of the pyloroplasty pouches. In difficult cases it may be valuable to repeat the examination after a few months when changes in the postoperative deformity will indicate continued ulceration and fibrosis and may show at that time an ulcer crater.

\section{Results}

The patients will be divided into two groups: those in whom an ulcer has been proved by a second operation and those in whom there is no proof of recurrent ulceration.

\section{PATIENTS WITH PROVEN RECURRENT} ULCERATION

Twelve patients show strong evidence of a recurrent ulcer. Two have since had transthoracic vagotomy for incomplete nerve section with subsequent disappearance of both symptoms and radiological abnormality, while the other 
10 have had the recurrent ulcer demonstrated at the second operation. Reoperation consisted of repeating the vagotomy and pyloroplasty in one patient and performing an antrectomy in the nine others. The antrectomy was accompanied by a repeat abdominal vagotomy in five patients and in eight the anastomosis was gastroduodenal. With the exception of the patient having a repeat vagotomy and pyloroplasty all are now cured of their symptoms.

\section{Radiological assessment}

The radiologist's report made at the time of the investigation makes a diagnosis of recurrent ulcer in eight out of the 12 patients. However, with the cumulative experience of the entire series and after consideration of the normal findings after vagotomy reported in a previous paper (Toye et al, 1970) it has become possible to revise these earlier reports. We therefore set up a 'blind' assessment of the films seen without knowledge of the patient's name and it was possible to detect an ulcer crater in 10 and stenosis in five. In all 12 patients either one or the other abnormality was present.

Examples of the radiographs showing recurrent ulcer are seen in Figures 1 and 2.

PATIENTS WITHOUT PROVEN RECURRENCE Twelve patients with recurrent dyspepsia have been studied and followed from two to seven years after their first operation.

Four patients on suspicion of recurrent ulceration have had transthoracic vagotomy for incomplete nerve section and in only one of these has there been any improvement. Three of these four have also had cholecystectomies for cholelithiasis but this procedure has relieved only one of these patients of his symptoms.

Eight patients have had no further operation since their investigation for recurrent dyspepsia. In three the symptoms have now disappeared, in two the symptoms have become more clearly those of oesophagitis and this diagnosis was substantiated by the barium meal findings and confirmed in two by oesophageal perfusion (Bennett and Atkinson, 1967). The remaining five patients have been reviewed for three to five years and have not developed a pattern of symptoms typical of peptic ulceration nor have they produced any complication. At least three patients have evidence to suggest hypochondriasis and two of these had no macroscopical evidence of duodenal ulcer at the original operation. In none of these patients can we prove that there has been no recurrence of their peptic ulcer after vagotomy and pyloroplasty. However, with the one exception of the patient who improved after transthoracic vagotomy (with no change in the $x$-ray appearances), we think there is strong circumstantial evidence that these 12 patients have not had a recurrent ulcer.

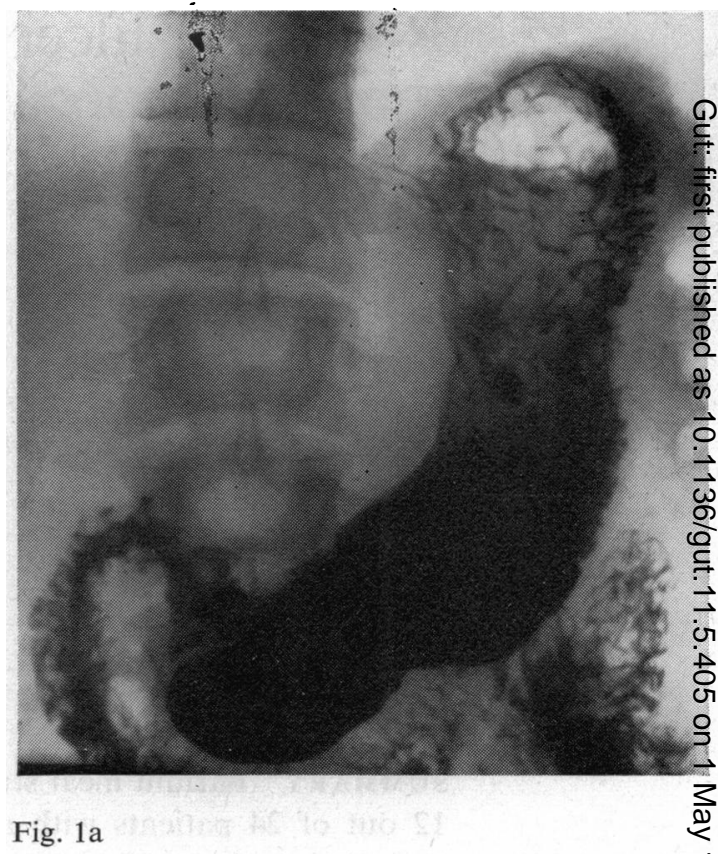

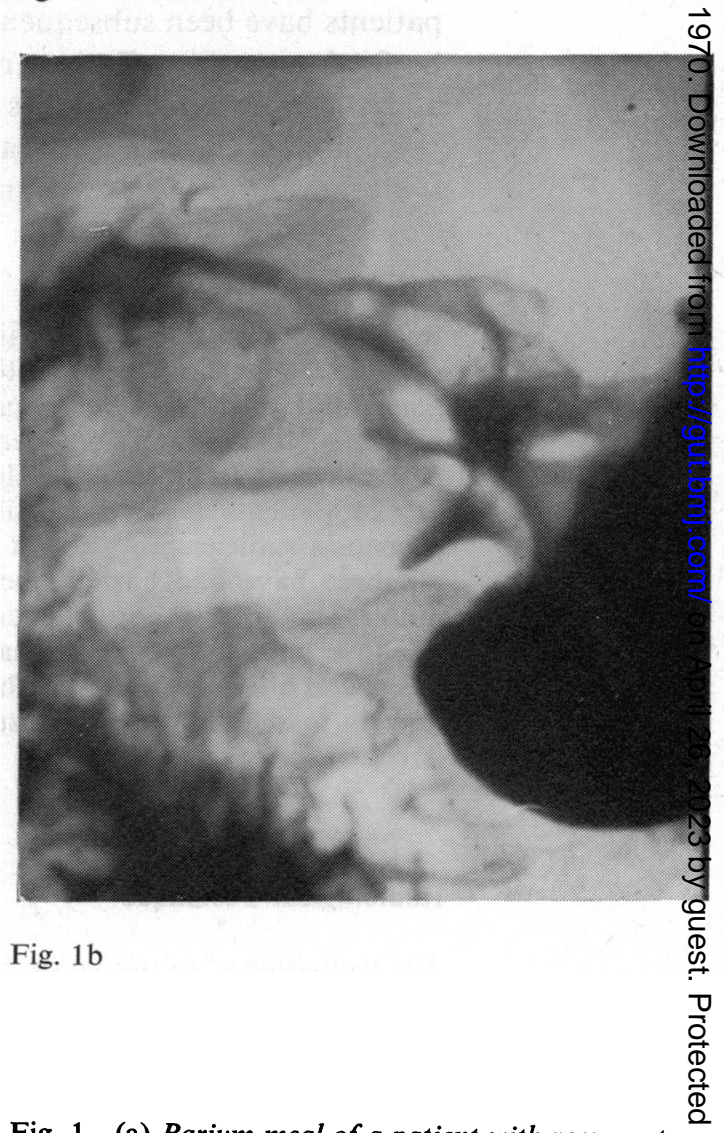

Fig. 1 (a) Barium meal of a patient with recurrent symptoms after vagotomy and pyloroplasty, demonstrating normal gastric emptying with an apparently wide channel; (b) and (c) mucosal studies showing a large recurrent ulcer in the pyloric channel; (d) oneō of a series of mucosal studies in the same patient after transthoracic vagotomy, showing that the ulcer crater has disappeared. 


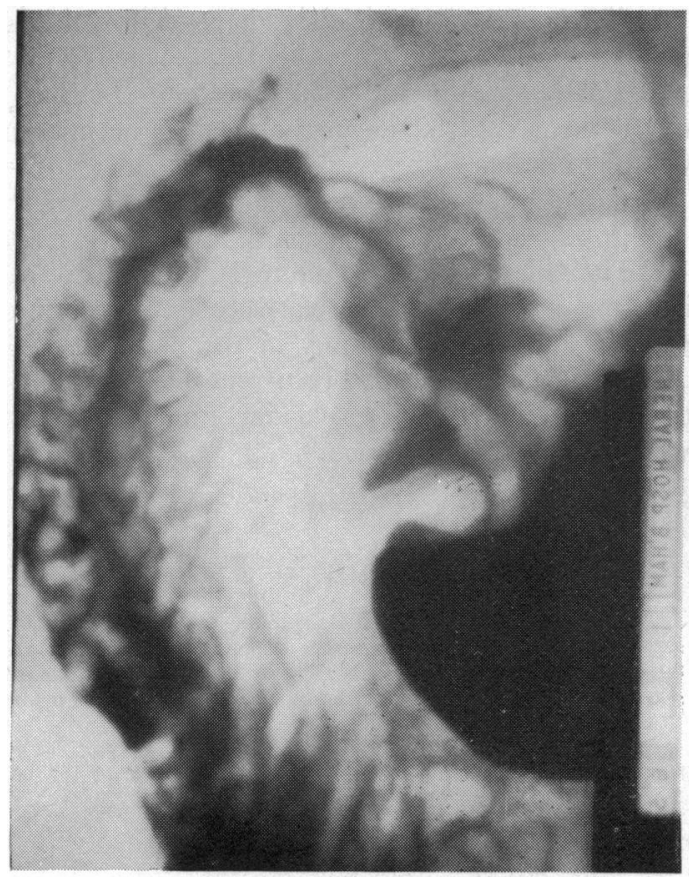

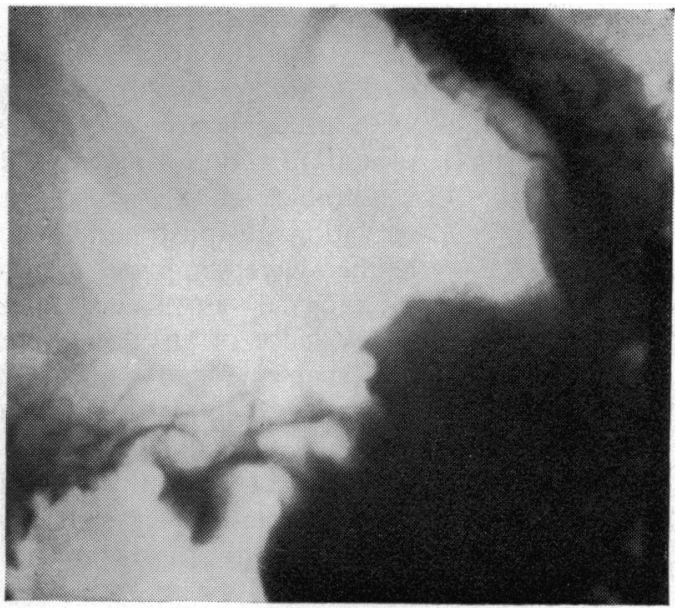

Fig. 1d

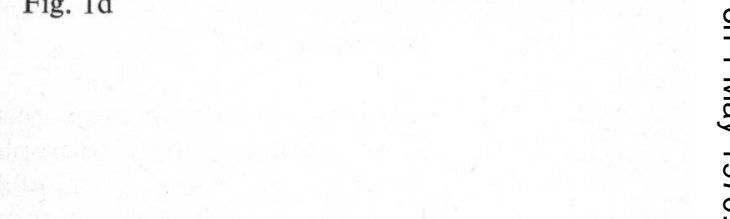

Fig. 1c

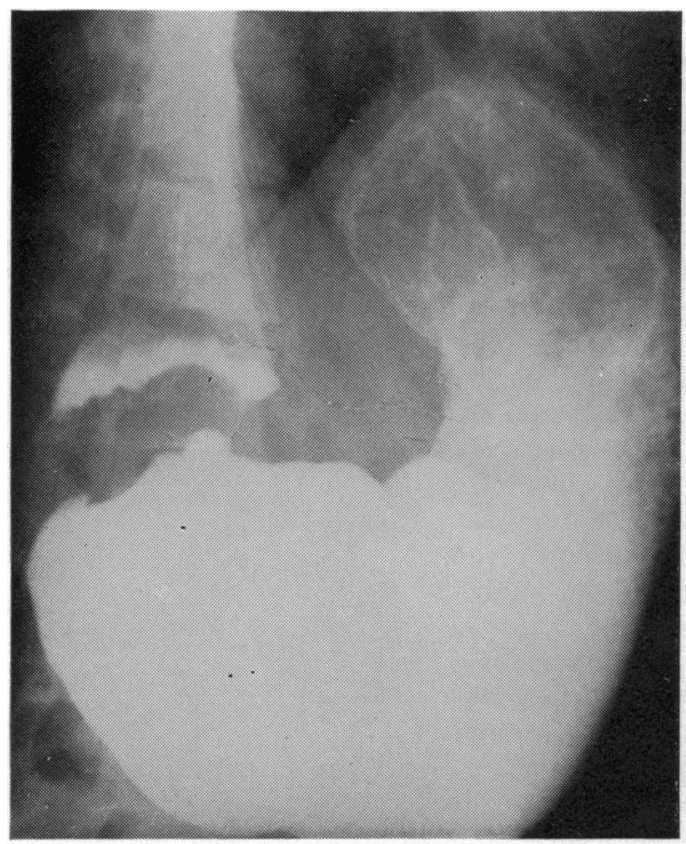

Fig. 2a

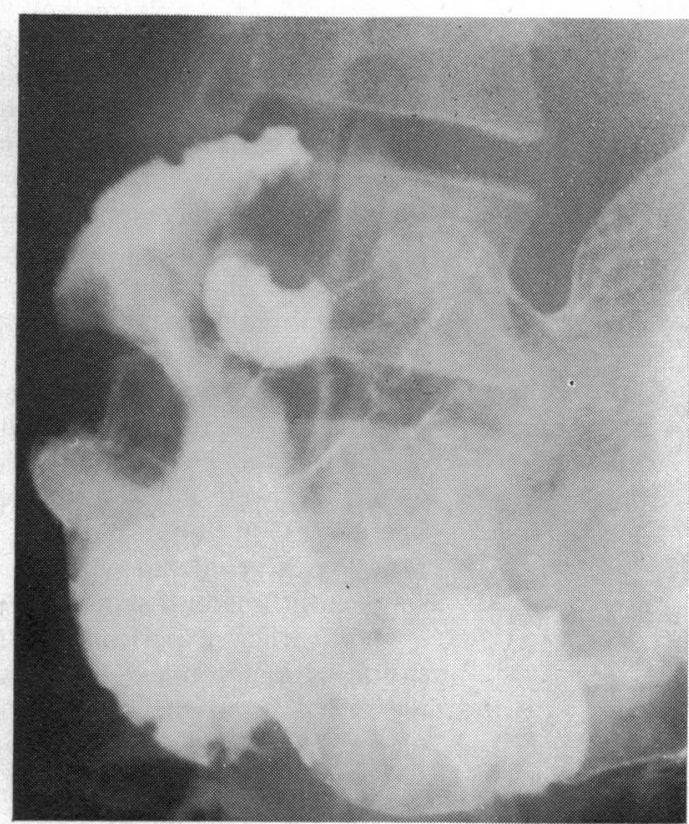

Fig. 2b

Fig. 2 (a) Grossly distended stomach with residue.

(b) Ulcer crater situated proximal to obstruction in the pyloroplast channel. (This patient was subsequently cured by antrectomy.)

Reprinted from 'After Vagotomy', edited by Williams and A. G. Cox, Butterworths, London, by permission of the publishers. 


\section{Radiological assessment}

In two of these 12 patients the postoperative barium studies performed for recurrent dyspepsia were originally reported as showing recurrent ulceration. Both patients had transthoracic vagotomies as they also had incomplete denervation demonstrated by the insulin test and neither were improved as a result. The 'blind' radiological assessment was that there was no evidence of an ulcer crater in either patient and that one had some initial stenosis which was not present on a subsequent barium study. In one of the patients initially reported as having a large ulcer crater this is clearly distinguishable, in the light of later experience, as a normally emptying pyloroplasty pouch.

\section{Discussion}

In patients with proven recurrent ulceration after vagotomy and pyloroplasty it has been possible to make a confident radiological diagnosis providing that satisfactory films could be obtained. Confidence in the technique and interpretation has grown during the course of the experience of this study and in recent years the surgeon feels confident to advise reoperation in the presence of an ulcer crater or stenosis shown by a barium meal.

It is obviously more difficult to state confidently that there is no recurrence when the radiologist does not demonstrate any abnormal signs. In this series we have tended to 'beg the question', as one of our criteria for no recurrence has been the absence of $x$-ray evidence and some of our patients in the second group could still be proven to have recurrences at a later time. However, the evidence from barium meal studies provides a good practical guide in the management of recurrent dyspepsia, and surgeons would be well advised to hesitate before reoperating on any patient without positive radiological evidence and to repeat the study some months later if symptoms persist.

Our earlier experience with fruitless transthoracic vagotomies for suspected recurrent ulcers led us to the opinion that if a recurrent ulcer is suspected after vagotomy and drainage, it is better to re-explore through the abdomen and obtain positive proof of recurrence rather than resect the vagi through the chest. Of the six transthoracic vagotomies in this series only three relieved patients of their symptoms. This impression, however, may need re-appraisal in the light of the growing confidence in radiological interpretation. There may still be a place for the relatively simple operation of transthoracic vagotomy in the patient with a clearly demonstrated recurrent ulcer, a freely draining stomach, and proven incomplete vagotomy.

References

Bennett, J. R., and Atkinson, M. (1966). The differentiation between oesophageal and cardiac pain. Lancet, 2, 1123-1127. Toye, D. K. M., Hutton, J. F., and Williams, J. A. (1970). Radiological evaluation after pyloroplasty. Gut, 11, 358-362. 